\title{
A mathematical formulation for integrated scheduling problem of handling equipment in container terminals
}

\author{
Yan Zheng ${ }^{1}$, Yujie Xiao ${ }^{2}$ \\ ${ }^{1}$ College of Automobile and Traffic Engineering, Nanjing Forestry University, Nanjing, 210037, China \\ ${ }^{2}$ School of Marketing and Logistic Management, Nanjing University of Finance and Economics, \\ Nanjing, 210046, China
}

Keywords: Integrated Scheduling, Container Terminals, Mixed Integer Linear Programming.

\begin{abstract}
This paper studies an integrated scheduling problem of quay cranes, yard trucks and yard cranes in container terminals. As each container is sequentially handled by these three interrelated handling equipment, the global optimal solution may not be guaranteed by only considering one type of equipment separately. The proposed problem considers the interdependency of different handling equipment to improve the overall performance of container terminals. In this paper, a mixed integer linear programming is proposed to search the optimal solution for this integrated problem. The results of computational experiments show that the integrated scheduling problem in small size could be efficiently solved by using the mathematical model.
\end{abstract}

\section{Introduction}

The globalization of trade has facilitated the development of worldwide logistics and transportation systems, especially the maritime container terminals (CTs). The ship turnaround time which is the total time spent by a ship at berth is one critical indicator for CT's efficiency [1]. Terminals with shorter ship turnaround time can keep up with the competition and hold a leading position in the world. To achieve this, the close cooperation of activities in CTs is required.

In CTs, three types of handling equipment are mainly deployed to handle containers [2]. They are quay cranes (QCs), yard trucks (YTs) and yard cranes (YCs). QCs are located in the quayside area and responsible for loading/unloading containers onto/from the ship. YCs serve in the storage yard to lift/place containers from/onto YTs and stack or restack containers in the block. YTs are used to transport containers between QCs and YCs. This paper is to study an integrated model of three handling equipment QCs, YTs and YCs, named i_QCYTYC. The objective is to minimize the makespan, which directly reflects the ship turnaround time.

As stated by Zeng \& Yang [3], most CTs employ the methods where loading and unloading are scheduled, respectively. In this paper, loading operations of outbound containers are taken into account. QCs assignment to ships has already been determined. According to the type of containers and the places where containers are shipped, the location of each container in the ship is also predetermined. That is, each container has a predefined QC. The sequence of containers on each QC is to be determined. Besides the assignment of YTs, the assignment of YCs to blocks is considered to fully utilize YCs and reduce potential investment cost [4]. Thus, the aim of i_QCYTYC is to concurrently make decisions on three related sub-problems: the loading sequence of each QC, dispatching YTs to containers and dispatching YCs to containers. To find the optimal solution to the proposed integrated scheduling problem, a mixed integer linear programming (MILP) is developed to find the optimal solution for i_QCYTYC.

\section{Problem Description and Mathematical Formulation}

Figure 1 shows a typical layout of a CT. Ships berth along the seaside and they are served by a number of QCs. The storage yard is separated into several blocks. Transfer points are located in front of each QC and each block. YTs receive/discharge containers at the transfer points. For outbound 
containers, three stages are involved to complete the process of loading containers onto ships. First, a set of YCs moves inside the storage area to pick up the required containers from blocks and load them onto YTs. Then, YTs are dispatched to transport the containers to QCs. Finally, QCs load the containers onto the ships. Each YT or YC begins to work from its initial position. The distance between any two transfer points is predetermined.

In i_QCYTYC, a job is referred to as a complete transferring process for a container. The origin and destination of a container are the block and QC, respectively. The assigned block and QC for each container as well as the initial positions for YTs and YCs are given. The assumptions adopted for i_QCYTYC are introduced as follows: a) identical YTs/YCs are used in CTs; b) loaded YTs/YCs and empty YTs/YCs are assumed to move at the same speed; c) YTs have the unit capacity; d) the handling time of QCs/YCs for containers is assumed to be the average handling time; e) special containers are not included. All containers can be stacked on the top of each other; f) control problems such as traffic congestion and conflicts of YTs and YCs are not in the consideration of this study.
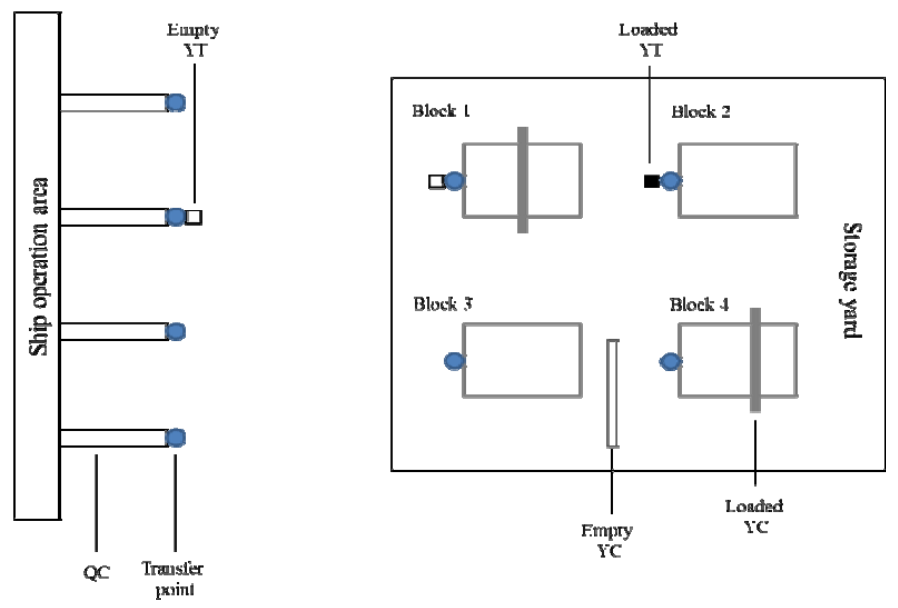

Figure 1 A typical layout of a CT

The result of i_QCYTYC is composed of a detailed schedule for QCs, YTs and YCs, including: (1) the start times and the finish times of each job on QC, YT and YC, (2) the sequence of jobs on each QC, YT and YC, and (3) YTs assignment as well as YCs assignment. The objective of i_QCYTYC is to minimize the makespan.

The following notations are used to describe i_QCYTYC in this paper:

Index:

$\mathrm{i}, \mathrm{j}$ Indices for jobs, $\mathrm{i}, \mathrm{j}=1,2, \ldots, \mathrm{Njob}, \mathrm{Njob}=$ the number of jobs

$\mathrm{q}$ Index for QCs, $\mathrm{q}=1,2, \ldots, \mathrm{Nqc}, \mathrm{Nqc}=$ the number of $\mathrm{QCs}$

$\mathrm{k}$ Index for YTs, $\mathrm{k}=1,2, \ldots, \mathrm{Nyt}, \mathrm{Nyt}=$ the number of YTs

e Index for YCs, e $=1,2, \ldots, N y c, N y c=$ the number of $\mathrm{YCs}$

$\mathrm{m}, \mathrm{n} \quad$ Indices for locations of QCs and blocks

Parameters:

QC(i) QC assigned to job i

$\mathrm{B}(\mathrm{i})$ Block where job i is stored

iptk/ ipce Initial position of YT k/YC e

Dmn Distance from location $\mathrm{m}$ to location $\mathrm{n}$

hqc/hyc Average handling time spent by a QC/YC

syt/syc Moving speed of YTs/YCs

M A very large positive constant

Sets:

L Set of outbound containers

SQ Set of QCs

ST Set of YTs 
SC Set of YCs

SPq Set of pairs of jobs i and j, i.e. (i,j) where $Q C(i)=Q C(j)=q$

Decision variables:

Cmax Makespan

Tyti Arrival time of job $i$ at the transfer point of QC(i) by its assigned YT

Tyci Arrival time of job $i$ at the transfer point of B(i) by its assigned YC

ci Completion time of job i, i.e. the finish time of job $\mathrm{i}$ on QC(i) and the start time of job i on $\mathrm{QC}(\mathrm{i})$ is (ci - hqc)

$u_{i k}=\left\{\begin{array}{l}1, \text { if job } i \text { is assigned to YT } k \\ 0, \text { otherwise. }\end{array}\right.$

$v_{i e}=\left\{\begin{array}{l}1, \text { if job } i \text { is assigned to YC } e \\ 0, \text { otherwise. }\end{array}\right.$

$x_{i j q}=\left\{\begin{array}{l}1, \text { if job } i \text { precedes job } j \text { on } Q C q \text { and }(i, j) \in S P_{q} \\ 0, \text { otherwise. }\end{array}\right.$

$y_{i j k}=\left\{\begin{array}{l}1, \text { if job } i \text { is immediately before job } j \text { on YT } k \\ 0, \text { otherwise. }\end{array}\right.$

$Z_{i j e}=\left\{\begin{array}{l}1, \text { if job } i \text { is immediately before job } j \text { on YC } e \\ 0, \text { otherwise. }\end{array}\right.$

$f 1_{i k}=\left\{\begin{array}{l}1, \text { if job } i \text { is the first task of YT } k \\ 0, \text { otherwise. }\end{array}\right.$

$f 2_{i e}=\left\{\begin{array}{l}1, \text { if job } i \text { is the first task of YCe } \\ 0, \text { otherwise. }\end{array}\right.$

The MILP model for i_QCYTYC is given as follows. The objective function is to minimize the makespan.

Minimize Cmax

$$
\begin{gathered}
C_{\max } \geq c_{i}, \forall i \in L \\
c_{i}-h q c \geq T y t_{i}, \forall i \in L \\
T y t_{i}-D_{B(i) Q C(i)} / s y t \geq T y c_{i}, \forall i \in L \\
T y c_{j}-h y c+M\left(1-z_{i j e}\right) \geq T y t_{i}-D_{B(i) Q C(i)} / s y t+D_{B(i) B(j)} / s y c, \forall i, j \in L, \forall e \in S C \\
T y t_{j}-D_{B(j) Q C(j)} / s y t+M\left(1-y_{i j k}\right) \geq c_{i}-h q c+D_{Q C(i) B(j)} / s y t, \forall i, j \in L, \forall k \in S T \\
T y t_{i}+M\left(1-f 1_{i k}\right) \geq\left(D_{i p t_{k} B(i)}+D_{B(i) Q C(i)}\right) / s y t, \forall i \in L, \forall k \in S T \\
T y c_{i}+M\left(1-f 2_{i e}\right) \geq D_{i p c_{e} B(i)} / s y C+h y c, \forall i \in L, \forall e \in S C \\
c_{j}-c_{i}+M\left(1-x_{i j q}\right) \geq h q c, \forall(i, j) \in S P_{q}, \forall q \in S Q \\
c_{i}-c_{j}+M x_{i j q} \geq h q c, \forall(i, j) \in S P_{q}, \forall q \in S Q \\
\sum_{k=1}^{N y t} u_{i k}=1, \forall i \in L \\
\sum_{e=1}^{N y c} v_{i e}=1, \forall i \in L \\
u_{i k}+u_{j k}-2 y_{i j k} \geq 0, \forall i, j \in L, \forall k \in S T \\
v_{i e}+v_{j e}-2 z_{i j e} \geq 0, \forall i, j \in L, \forall e \in S C \\
\sum_{i=1}^{N j o b} f 1_{i k}=1, \forall k \in S T
\end{gathered}
$$




$$
\begin{aligned}
& \sum_{i=1}^{N j o b} f 2_{i e}=1, \forall e \in S C \\
& f 1_{i k}-u_{i k} \leq 0, \forall i \in L, \forall k \in S T \\
& f 2_{i e}-v_{i e} \leq 0, \forall i \in L, \forall e \in S C \\
& \sum_{k=1}^{N y t} y_{i j k}+\sum_{k=1}^{N y t} y_{j i k} \leq 1, \forall i, j \in L \\
& \sum_{k=1}^{N y t}\left(f 1_{i k}+\sum_{\substack{\forall j \in L \\
i \neq j}} y_{j i k}\right)=1, \forall i \in L \\
& \sum_{k=1}^{N y t} \sum_{\substack{\forall \in L \\
i \neq j}} y_{i j k} \leq 1, \forall i \in L \\
& \sum_{e=1}^{N y c} z_{i j e}+\sum_{e=1}^{N y c} z_{j i e} \leq 1, \forall i, j \in L \\
& \sum_{e=1}^{N y c}\left(f 2_{i e}+\sum_{\substack{\forall j \in L \\
i \neq j}} z_{j i e}\right)=1, \forall i \in L \\
& \sum_{e=1}^{N y c} \sum_{\substack{\forall j \in L \\
i \neq j}} z_{i j e} \leq 1, \forall i \in L \\
& x_{i j q}, u_{i k}, v_{i e}, y_{i j k}, z_{i j e}, f 1_{i k}, f 2_{i e} \in\{0,1\}, \forall i, j \in L, \forall q \in S Q, \forall k \in S T, \forall e \in S C
\end{aligned}
$$

Constraint (1) defines the makespan. Constraint (2) calculates the completion time of each job and implies that each job can be handled by a QC after this job has been transferred to the transfer point of this QC by a YT. Constraint (3) makes sure that each job can be handled by a YT after this job has been transferred to the transfer point of the block by a YC. Constraints (4a), (4b) define the blocking constraints. Constraints (5a), (5b) force each YT and each YC to start its first task from its initial position, respectively. Constraints (6a), (6b) ensure that a pair of jobs (i, j) on the same QC q will not be handled simultaneously. Constraints (7a), (7b) ensure that each job is assigned to only one YT and only one YC, respectively. Constraint (8a) describes that if jobs $\mathrm{i}$ and $\mathrm{j}$ are sequentially handled by YT k (i.e. yijk = 1), jobs i and j must be assigned to YT k (i.e. uik = 1 and ujk = 1). Likewise, constraint (8b) is imposed on YCs. Constraints (9a), (9b) determine the first task for each YT and each YC, respectively. Constraint (10a) describes that if job $\mathrm{i}$ is the first task of YT k, job i must be assigned to YT k. Constraint (10b) describes that if job i is the first task of YC e, job i must be assigned to YC e. Constraints (11a)-(11c) determine the sequence of jobs on each YT. Constraints (12a)-(12c) determine the sequence of jobs on each YC. Constraint (13) defines the binary variables.

\section{Computational Experiments}

To evaluate the performance of the proposed GA, seven (e1-e7) problem instances are generated based on the data from the published literature [5, 6]. In the layout of CT adopted for the analysis, there are six positions of QCs and 20 blocks in the storage yard. The assigned QC and block for each job is randomly generated. The initial positions of YTs and YCs are created in a random way. The moving speed of YTs and YCs is assumed to be $4 \mathrm{~m} / \mathrm{s}$ and $3 \mathrm{~m} / \mathrm{s}$, respectively. The distance between any two locations of QCs and blocks is assumed to be along the shortest path. The average handling time of QCs is assumed to be 60 seconds. The average handling time of YCs is assumed to be 100 seconds. The numbers of jobs, QCs, YTs and YCs in seven problem instances are shown in Table I.

Table II presents the results of the seven computational experiments obtained by the proposed MILP. For the small-sized problems e1 and e2, the optimal solutions are found by MILP. As other problem instances cannot be solved to optimality within the reasonable computation time, the running 
time of CPLEX is limited to 36 hours. The best obtained solution within 36 hours is recorded for MILP. From Table II, it can be seen that MILP is intractable to be solved with the increase of problem size. Therefore, it is necessary to develop an efficient heuristic algorithm to solve the integrated scheduling problem in realistic world.

Table 1 Details of seven problem instances

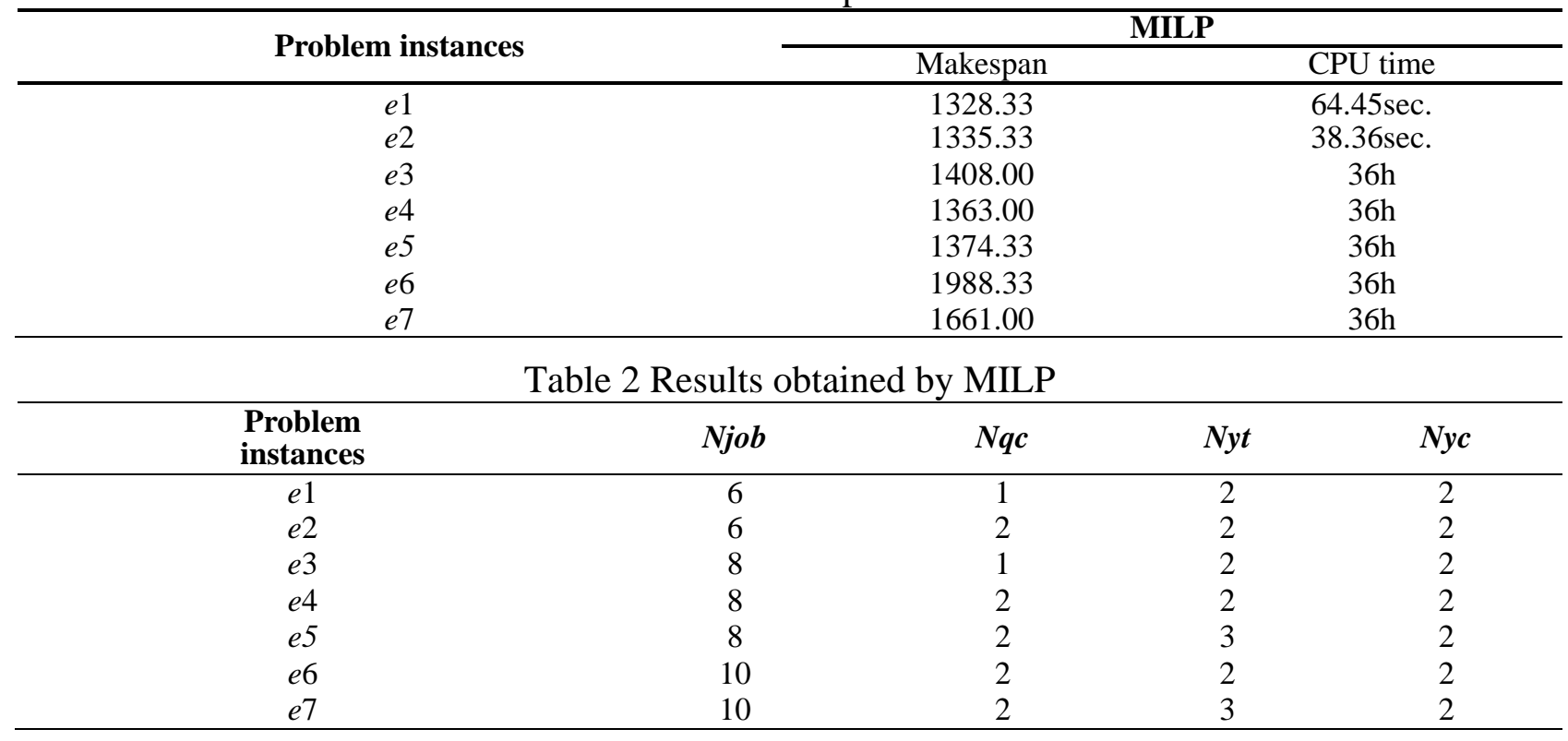

\section{Conclusion}

Since optimizing only one type of handling equipment may not guarantee the global optimal solution, the purpose of this paper is to study the integrated scheduling problem of three interconnected handling equipment QCs, YTs and YCs in CTs. In this paper, an MILP model is presented to find the optimal solution. As the unloading operation is processed in the reverse order of the loading operation, the proposed solution methods can also be applied to unloading operations of inbound containers. In the future, the research topic on the application of the heuristic algorithm to the integrated problems will be studied. The integrated model and the developed solution method of this paper can be extended by considering more practical constraints, such as the ready time and the due date of each container, the storage space allocation, etc.

\section{Acknowledgements}

Dr. Yujie Xiao is the corresponding author. The research has been supported by the National Natural Science Foundation of China (Grant No. 71701099, 71501090) and by the Natural Science Foundation of Jiangsu Provincial Universities (Grant No. 17KJB580008).

\section{References}

[1]. Chen, L., Bostel, N., Dejax, P., Cai, J. and Xi, L., 2007. A tabu search algorithm for the integrated scheduling problem of container handling systems in a maritime terminal. European Journal of Operational Research, 181(1), 40-58.

[2]. Steenken, D., Voß, S. and Stahlbock, R., 2004. Container terminal operation and operations research - a classification and literature review. OR Spectrum, 26, 3-49.

[3]. Zeng, Q. and Yang, Z., 2009. Integrating simulation and optimization to schedule loading operations in container terminals. Computers \& Operations Research, 36, 1935-1944. 
[4]. He, J., Chang, D., Mi, W. and Yan, W., 2010. A hybrid parallel genetic algorithm for yard crane scheduling. Transportation Research Part E: Logistics and Transportation Review, 46(1), 136-155.

[5]. Cao, J.X., Lee, D-H., Chen, J.H. and Shi, Q., 2010. The integrated yard truck and yard crane scheduling problem: Benders' decomposition-based methods. Transportation Research Part E: Logistics and Transportation Review, 46(3), 344-353.

[6]. Lau, H.Y.K. and Zhao, Y., 2008. Integrated scheduling of handling equipment at automated container terminals. Annals of Operations Research, 159, 373-394. 